\title{
Status of the phenomena representation, 3D modeling, and cloud-based software architecture development
}

\author{
Curtis Smith \\ Steven Prescott \\ Kellie Kvarfordt \\ Ram Sampath \\ Katie Larson
}

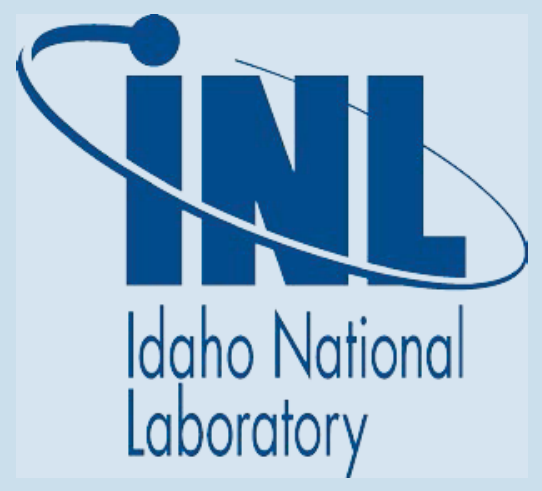

September 2015

DOE Office of Nuclear Energy 


\section{DISCLAIMER}

This information was prepared as an account of work sponsored by an agency of the U.S. Government. Neither the U.S. Government nor any agency thereof, nor any of their employees, makes any warranty, expressed or implied, or assumes any legal liability or responsibility for the accuracy, completeness, or usefulness, of any information, apparatus, product, or process disclosed, or represents that its use would not infringe privately owned rights. References herein to any specific commercial product, process, or service by trade name, trade mark, manufacturer, or otherwise, do not necessarily constitute or imply its endorsement, recommendation, or favoring by the U.S. Government or any agency thereof. The views and opinions of authors expressed herein do not necessarily state or reflect those of the U.S. Government or any agency thereof. 


\title{
Status of the phenomena representation, 3D modeling, and cloud-based software architecture development
}

\author{
Curtis L Smith \\ Steven Prescott \\ Kellie Kvarfordt \\ Ram Sampath \\ Katie Larson
}

September 2015

Idaho National Laboratory

Idaho Falls, Idaho 83415

Prepared for the

U.S. Department of Energy

Office of Nuclear Energy

Under DOE Idaho Operations Office

Contract DE-AC07-05ID14517 


\section{SUMMARY}

Early in 2013, researchers at the Idaho National Laboratory outlined a technical framework to support the implementation of state-of-the-art probabilistic risk assessment to predict the safety performance of advanced small modular reactors. From that vision of the advanced framework for risk analysis, specific tasks have been underway in order to implement the framework. This report discusses the current development of a several tasks related to the framework implementation, including a discussion of a 3D physics engine that represents the motion of objects (including collision and debris modeling), cloud-based analysis tools such as a Bayesian-inference engine, and scenario simulations. These tasks were performed during 2015 as part of the technical work associated with the Advanced Reactor Technologies Program. 


\section{CONTENTS}

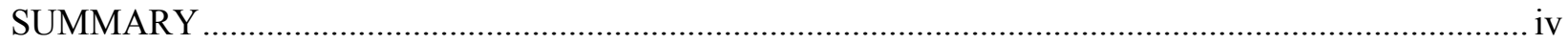

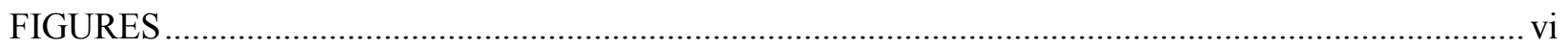

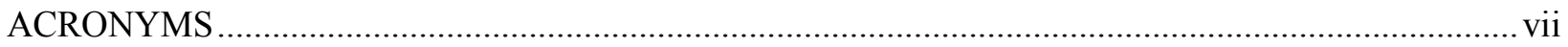

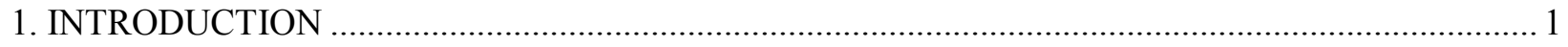

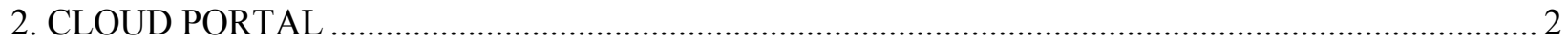

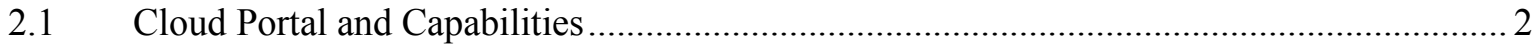

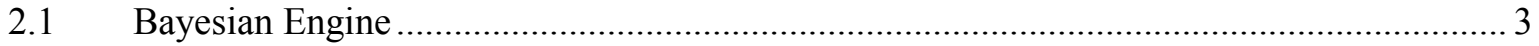

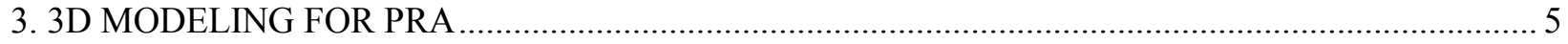

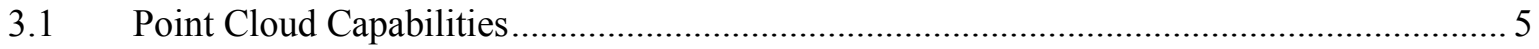

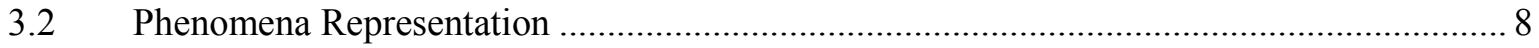

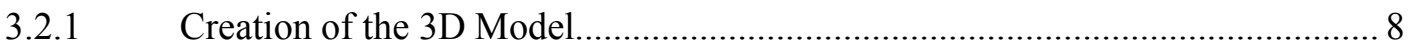

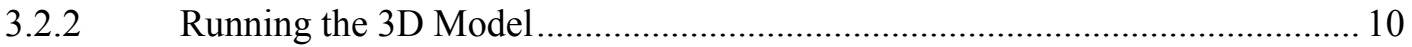

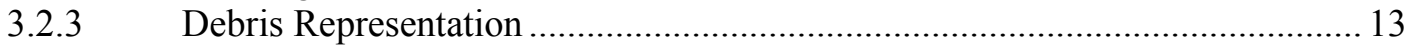

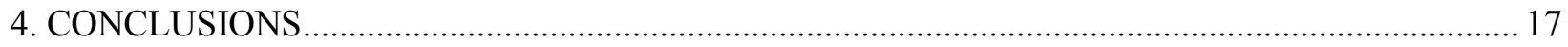

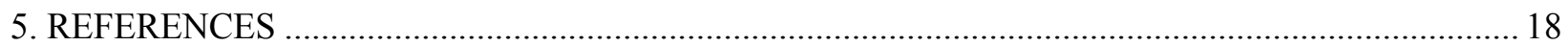




\section{FIGURES}

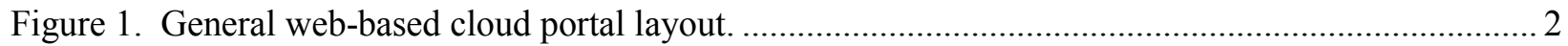

Figure 2. Examples of the types of tools found in the Safety Portal...................................................... 3

Figure 3: Visual OpenBUGS scripter accessibility approach............................................................ 4

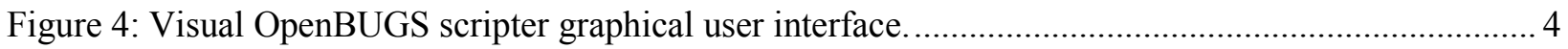

Figure 5. Point cloud representation for the generic cooling room.................................................... 6

Figure 6. Interior points are clustered (top) and then modeled (bottom)............................................. 7

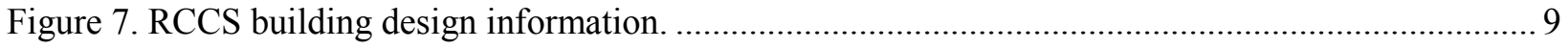

Figure 8. Placing the RCCS onto a hypothetical plant site ................................................................. 9

Figure 9: User interface for the Terrain Mesh Mapping tool............................................................... 10

Figure 10. SPH-based flooding example showing an external flood potentially impacting facility

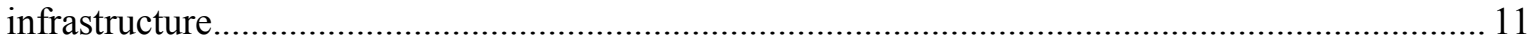

Figure 11. Force on the target facility infrastructure due to water impact............................................ 12

Figure 12. Height of water on the RCCS as a function of time during a tsunami scenario...................... 12

Figure 13. Progression of water on the RCCS during a tsunami scenario simulated using the 3D model

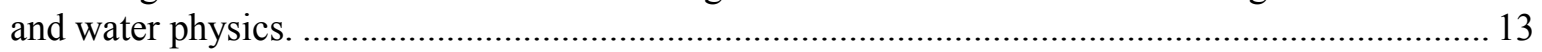

Figure 14. Representation of rigid bodies and debris in the SPH flood simulation................................ 14

Figure 15. Example of the dam breach scenario simulation............................................................. 15

Figure 16. SPH flood calculation showing the debris (truck) objects that may be moved during the

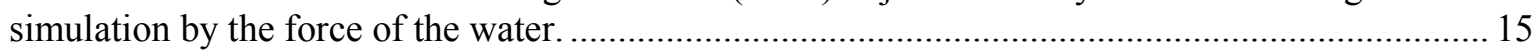

Figure 17. Example of the force and velocity information that is available to analysts as part of the flooding simulation. 


\section{ACRONYMS}

$\begin{array}{ll}\text { 3D } & \text { three-dimension } \\ \text { ART } & \text { Advanced Reactor Technologies } \\ \text { DOE } & \text { Department Of Energy } \\ \text { INL } & \text { Idaho National Laboratory } \\ \text { PRA } & \text { Probabilistic Risk Assessment } \\ \text { NRC } & \text { Nuclear Regulatory Commission } \\ \text { OpenBUGS } & \text { Open-source Bayesian inference Using Gibbs Sampling } \\ \text { PCL } & \text { Point Cloud Library } \\ \text { RCCS } & \text { reactor cavity cooling system } \\ \text { SMR } & \text { small modular reactor } \\ \text { SSC } & \text { systems, structures, and components }\end{array}$




\section{Cloud-based Architecture Capabilities}

\section{INTRODUCTION}

The Idaho National Laboratory (INL) has proposed research and development activities that address the formulation and development of a framework for applying modern computational tools to create advanced risk-based methods for identifying design vulnerabilities in advanced small modular reactors (SMRs). This framework will require the fusion of state-of-the-art probabilistic (PRA) methods, advanced 3D visualization methods, and high-performance computation within a flexible open source Framework. The work described in this report was produced as part of the technical research associated with the Advanced Reactor Technologies (ART) Program and describes the capabilities present in the cloud-based risk analysis portal and associated risk evaluation activities. The overall purpose of the cloud-based advanced SMR PRA Framework is to develop and demonstrate methodologies and tools that can be used to predict plant response and risk for safety application. The primary goal of the Framework is to provide a tool that can assist in future design and licensing activities by developing quantitative methods, tools, and the associated analysis framework for assessing a variety of SMR risks. We are addressing this goal within the ART Program by continuing the construction of a cloud-based analysis and modeling framework that will be used to demonstrate the technical basis related to SMR safety margins.

The objective of the work described in this report is to provide the status of implementing the Small Modular Reactor Detailed Technical Framework Specification (INL/EXT-13-28974), which details the specific activities necessary to establish the cloud-based PRA Framework to provide a structure for supporting modeling, phenomena representation, and risk integration/management via a cloud-based safety portal. (1)

This report is intended to present the 2015 status of several on-going advanced PRA tasks related to the framework implementation, including a discussion of a 3D physics engine that represents the motion of objects (including collision and debris modeling), cloud-based analysis tools such as a Bayesian-inference engine, and scenario simulations. These tasks were performed during 2015 as part of the technical work associated with the Advanced Reactor Technologies Program. These areas are addressed in two sections, Section 2 providing an overview of the cloud portal development and Section 3 providing the 3D modeling developments. 


\section{CLOUD PORTAL}

\subsection{Cloud Portal and Capabilities}

A cloud-based portal is a web application that integrates and manages a comprehensive collection of many different kinds of content including, but not limited to, web pages, web applications and documents where users may store, use, share, modify or otherwise contribute to projects. The portal maintains changes for all documents stored within the portal environment. It also maintains individual or a group of documents with role-based security access. Users are granted access based upon their need to know and are assigned to one or more security level groups. Within each security group, users have specific access to certain documents or application tools that are appropriate for that level.

The cloud portal accumulates many different kinds of information that are stored or serviced from different servers. These services (see Figure 1) include the Documents Server, Application Server, and Database Management Server. Specific applications may be developed to further summarize available information from various servers and present on the portal.

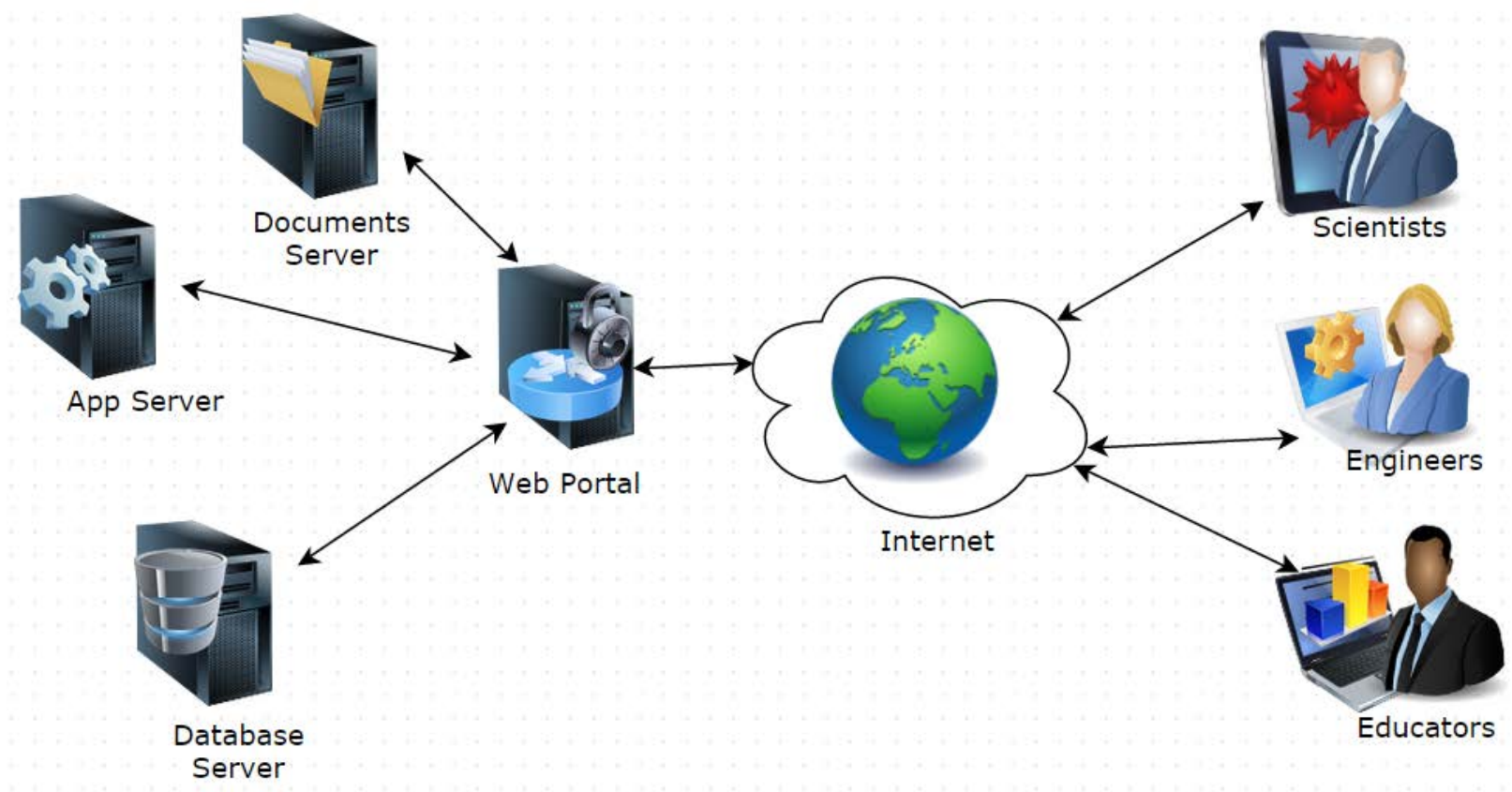

Figure 1. General web-based cloud portal layout.

The advanced PRA Safety Portal is being implemented as a cloud portal with emphasis on supporting PRA tools. In order to meet the needs of a variety of PRA tools that are or may be developed, design features were implemented to facilitate the types of common PRA tools that were anticipated for use in the portal. These features make it easier for existing tools to use the Safety Portal with minimal additions to existing code and for users to monitor changes to data they use. Additional details on the 
cloud portal design may be found in Reference (2). A summary of the types of attributes found in the Safety Portal are (with thumbnail screen shots shown in Figure 2):

- The portal has been created (found at safety.inl.gov) for use by the advanced SMR PRA community.

- All functionality runs via an internet web browser and has been developed in an integrated platform.

- Information sharing to facilitate having safety data available for analysts.

- Bayesian inference tool to perform probabilistic calculations.

- Terrain mapping to assist in creating 3D models.

- Fault tree solver (in progress) for system reliability analysis.

- Simulation tool (in progress) to represent scenarios for risk analysis.
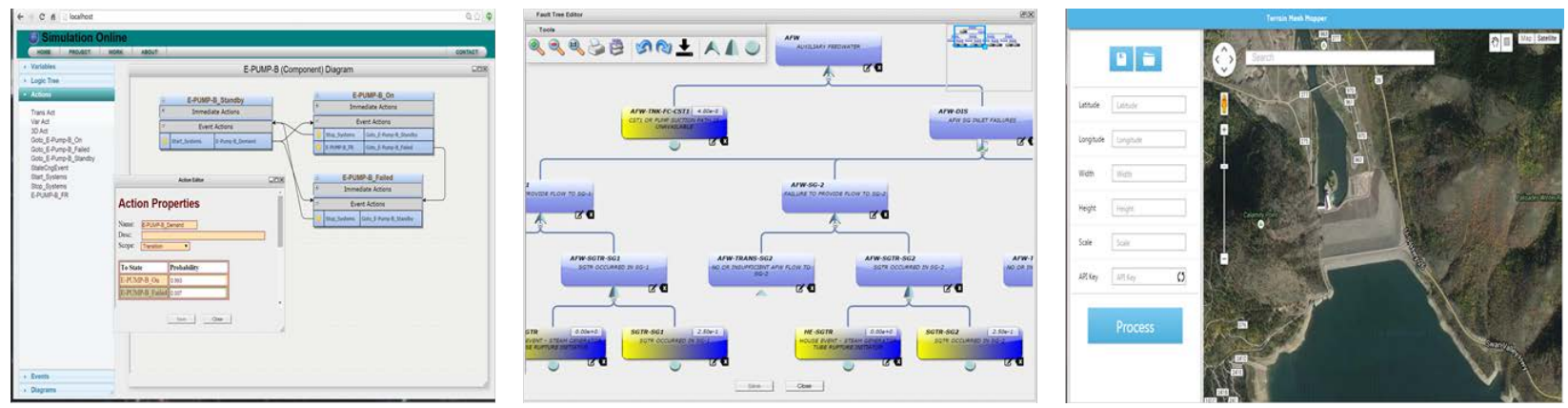

Figure 2. Examples of the types of tools found in the Safety Portal.

\subsection{Bayesian Engine}

The Bayesian Engine consists of an open-source software package called OpenBUGS (Opensource Bayesian inference Using Gibbs Sampling) that has been attached to a graphical user interface designed for the Safety Portal. This tool is designed to be flexible and enables analysts to have access to a shared high-performance computing environment for running models where normally such a highcomputing environment may not be readily available. The Bayesian software and its accessibility (see Figure 3) allows analysts with lower computing capability to visually create, modify, store and share probabilistic models via a drawing diagram of shapes representing software code such as OpenBUGS properties, functions, and procedures. The tool generates scripts based on the diagram, which are then submitted to a shared high-performance computer for execution (Figure 4). Status and progress reports are then relayed back to the analysts through the portal dashboard. 


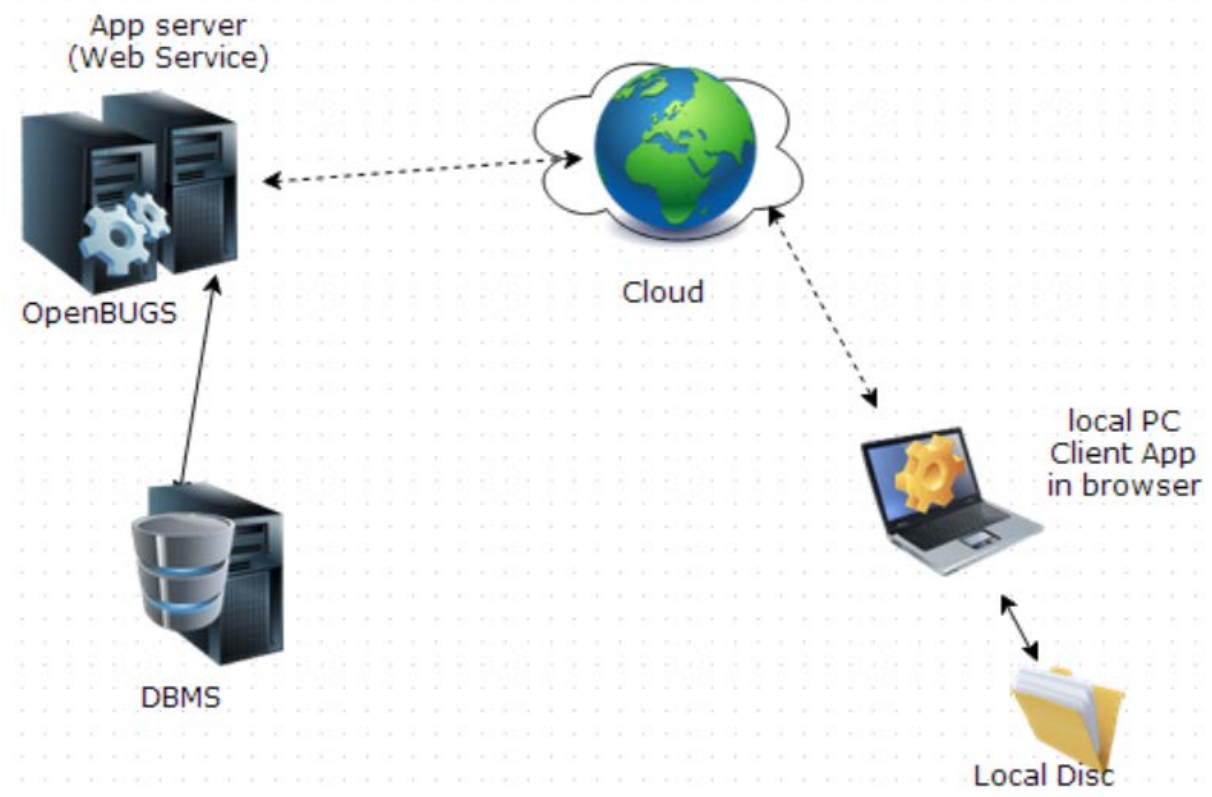

Figure 3: Visual OpenBUGS scripter accessibility approach.

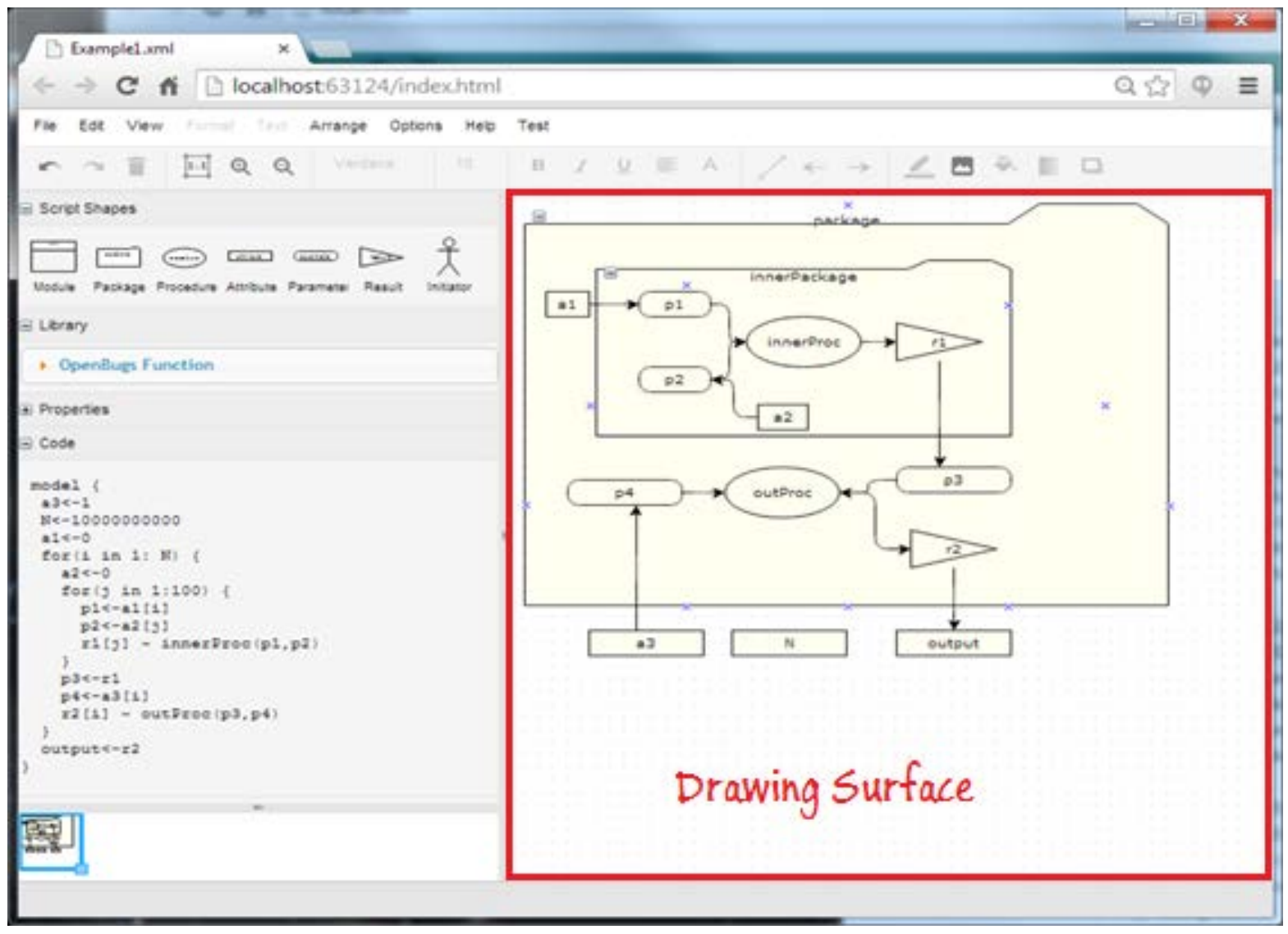

Figure 4: Visual OpenBUGS scripter graphical user interface. 


\section{3D MODELING FOR PRA}

\subsection{Point Cloud Capabilities}

One of the key features of the advanced PRA approach is in the use of 3D models to represent actual facilities and to provide a basis for simulation models. The risk analysis insights become more informative and valuable as the simulations demonstrate increased verisimilitude. This increase in realism encourages the use of detailed and accurate 3D models. However, manually creating these models may be a repetitive and time consuming task. It may also be difficult to obtain the many measurements required for high levels of detail for an already-built facility, as working off of design plans may be dated or may not include recent modifications.

Ideally, the process to create applicable 3D models could be automated to generate high-fidelity models more efficiently than through manual creation. Consequently, researchers have been investigating methods for automated model generation - much of the recent research proposes using "point clouds" as a foundation for 3D models. A "point cloud" is a set of data points in 3D space, where each point is defined by an $\mathrm{x}, \mathrm{y}$ and $\mathrm{z}$ coordinate. Point clouds can be generated in several different ways, but laser-based scanners are fast and offer the greatest accuracy. Top-of-the-line laser scanners can measure million points with an error of less than one millimeter.

One challenge to using point-clouds is in how to transform a given point cloud into a more usable polygon-based model. While this is still a research topic, current research has produced several algorithms and libraries to assist in the process. For example, the Point Cloud Library (PCL) is particularly useful. PCL is an open-source $\mathrm{C}++$ library with modules for cleaning, simplifying, analyzing, and editing point clouds. With these tools, it is possible to convert a point cloud of a complex structure or room into a useful model ready to be tested in a simulation. As an example, we used a point cloud produced at the INL of a generic building cooling room (see Figure 5). This point cloud contains information about the room such as size, shape, and location of objects. However, this information still needs to be turned into objects so that the 3D physics engine can interact with the room objects. 


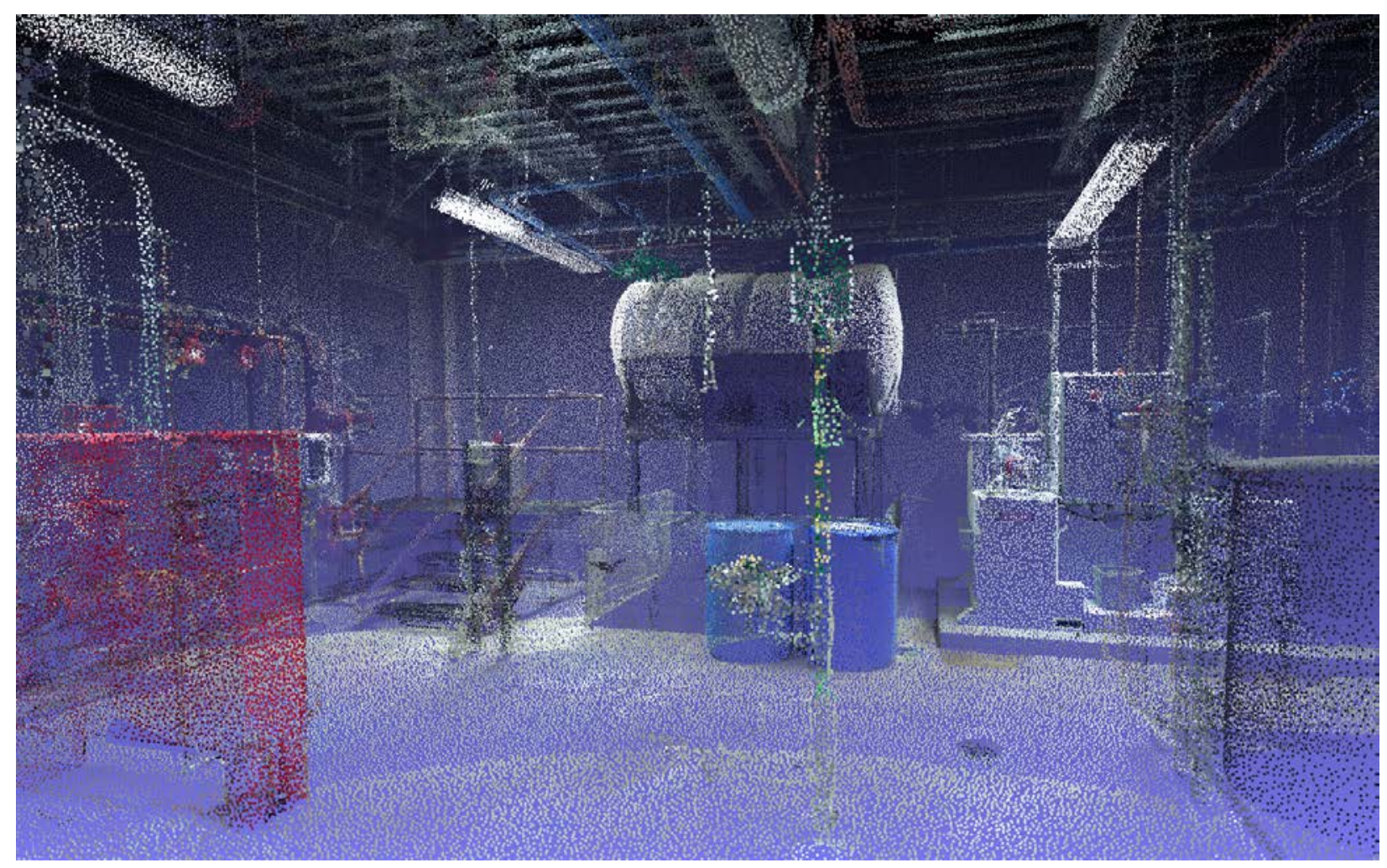

Figure 5. Point cloud representation for the generic cooling room.

To convert a point cloud to objects, several steps are performed: (1) noise removal, (2) downsampling the points to reduce the computational requirements, (3) surface reconstruction, (4) segregation of interior points from the wall points, and (5) manifold determination (i.e., solid object construction). Once completed, the 3D model needed to be "watertight" in that objects that are in reality solid need to be created such that there are no holes, discontinuities, or cracks. In the context of this application, watertight means that: (1) accidental holes created during surface reconstruction are filled and (2) realistic holes, such as an open window, are preserved. Upon completion of the process, we can extract the surfaces of the room model (see Figure 6). 


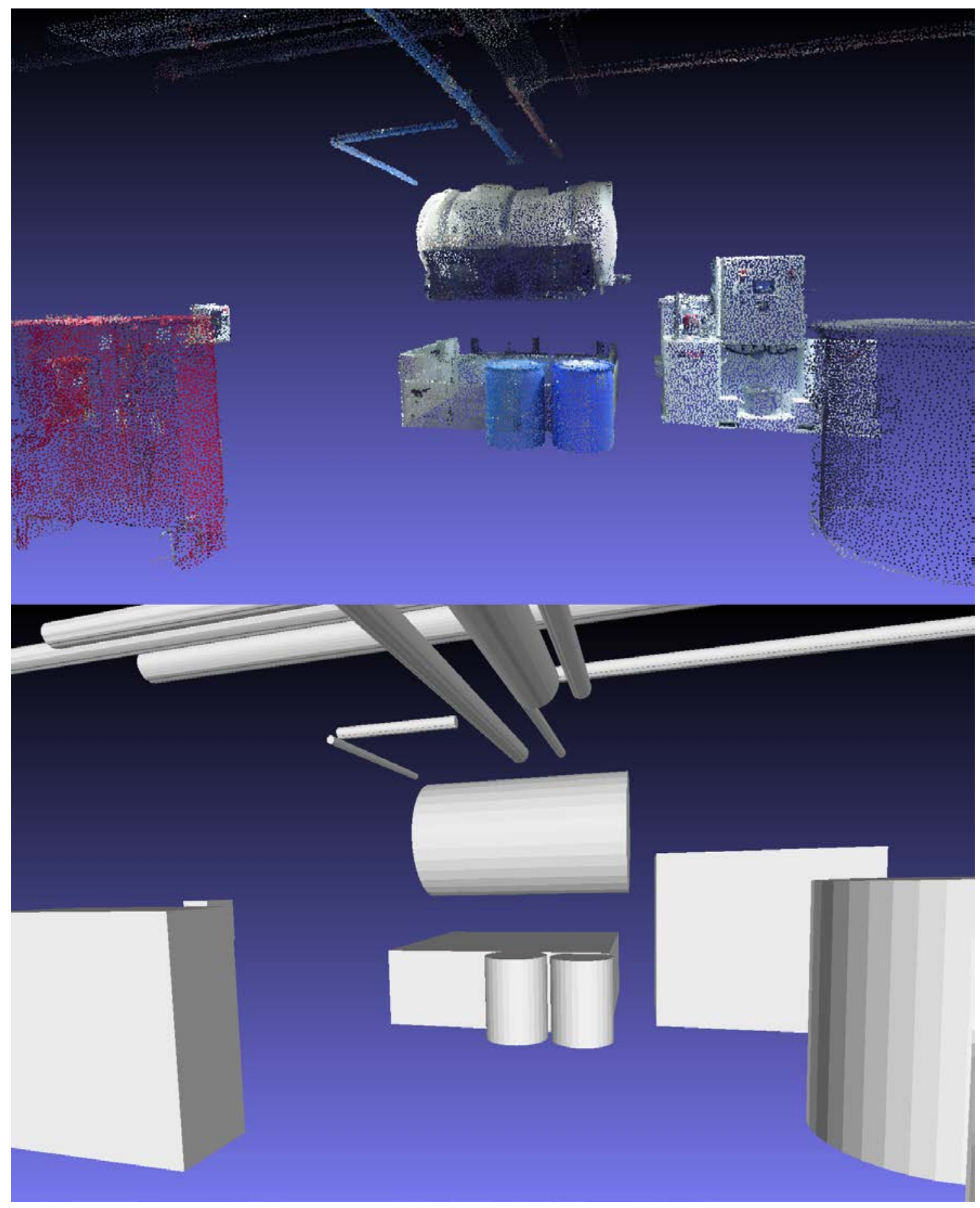

Figure 6. Interior points are clustered (top) and then modeled (bottom).

By using scanning equipment and automated algorithms we can quickly generate usable models for simulation. Following the automated generation process, additional "clean up" may need to be performed on the model, but the bulk of the model creation is captured via the automated-model step. 


\subsection{Phenomena Representation}

To represent accident scenarios in a simulation-based approach, we need to be able to describe and model specific types of physics. For example, to represent a flooding scenario, we need to be able to simulate water flows in and around a facility. For a risk analysis, we would like to have a single 3D representation of all key systems, structures, and components (SSCs) for the facility. Using this 3D model, we can simulate - by understanding how each SSC interacts with other parts of the facility (e.g., failure dependencies) - the hazard-induced susceptibilities of each SSC (e.g., energy from a seismic event may fail a component). This risk-based simulation requires two general steps, the creation of the 3D model and the representation of the desired physics using the 3D model.

\subsubsection{Creation of the 3D Model}

In order to create the 3D models to be used in the risk assessment scenarios, two general attributes need to be determined:

1. The facility SSCs represented via 3D objects.

2. The facility site topography represented as a 3D layout.

For facilities that already exist, the 3D models can be created manually (say from drawings) or may be generated using technology such as scanning and point clouds (see Section 3.1). For facilities that do not exist, the 3D model must be created from information such as computer-aided design files. As part of the work performed in 2015, we evaluated flooding for a facility that is currently in design, specifically the prototype facility being investigated at Argonne National Laboratory called the reactor cavity cooling system (RCCS). The RCCS is a passive system designed for heat removal.

Using the design information provided we created a 3D facility model of the RCCS in order to perform risk studies related to flooding. An example of the type of design information that was provided is shown in Figure 7. 

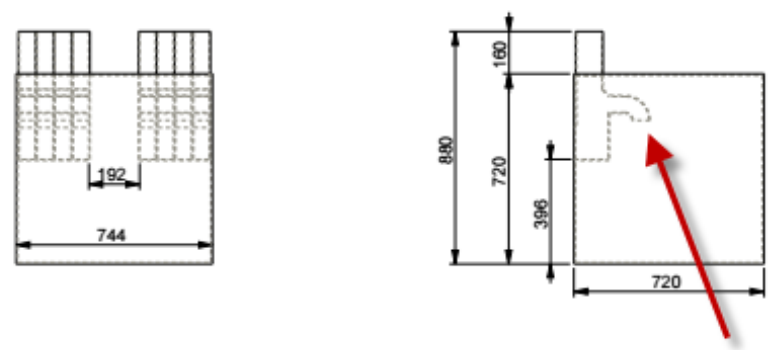

ALL DIMENSIONS IN INCHES
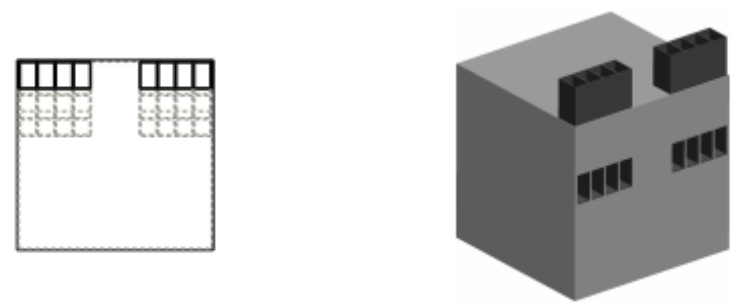

Figure 7. RCCS building design information.

The RCCS was placed on a hypothetical plant site (see Figure 8) in order to perform a risk analysis where we simulate a tsunami potentially inundating the plant site. In order to accurately represent water inundation onto the plant site, we needed to place the plant structures on a topography that may be representative of a location near the ocean. To create the topography information, we used the Terrain Mesh Mapper tool that is part of the Safety Portal. This tool allows a user to select an area on the Earth and convert it into a $3 \mathrm{D}$ polygon mesh representing the ground topography. A screen shot of the tool is shown in Figure 9.

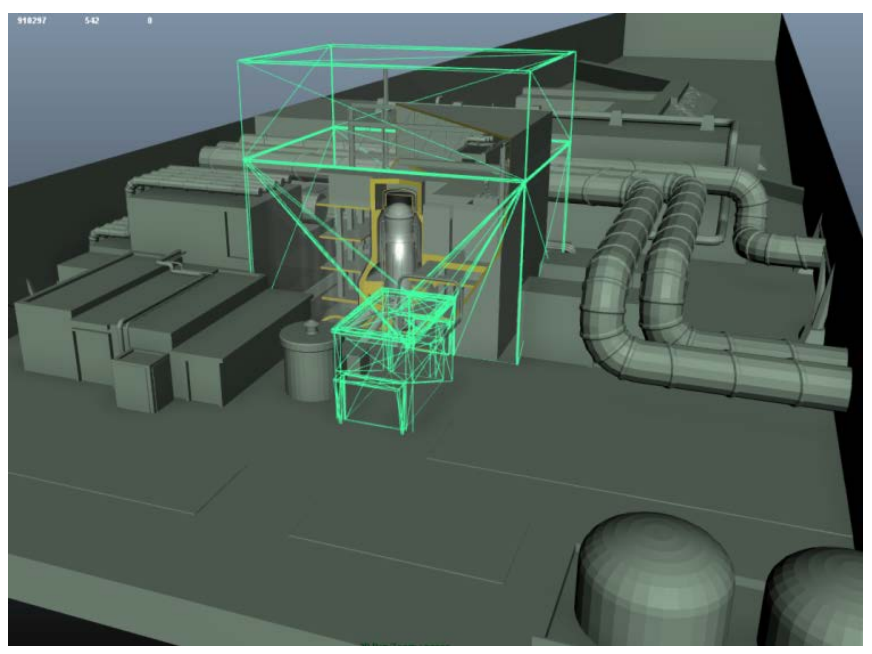

Figure 8. Placing the RCCS onto a hypothetical plant site. 


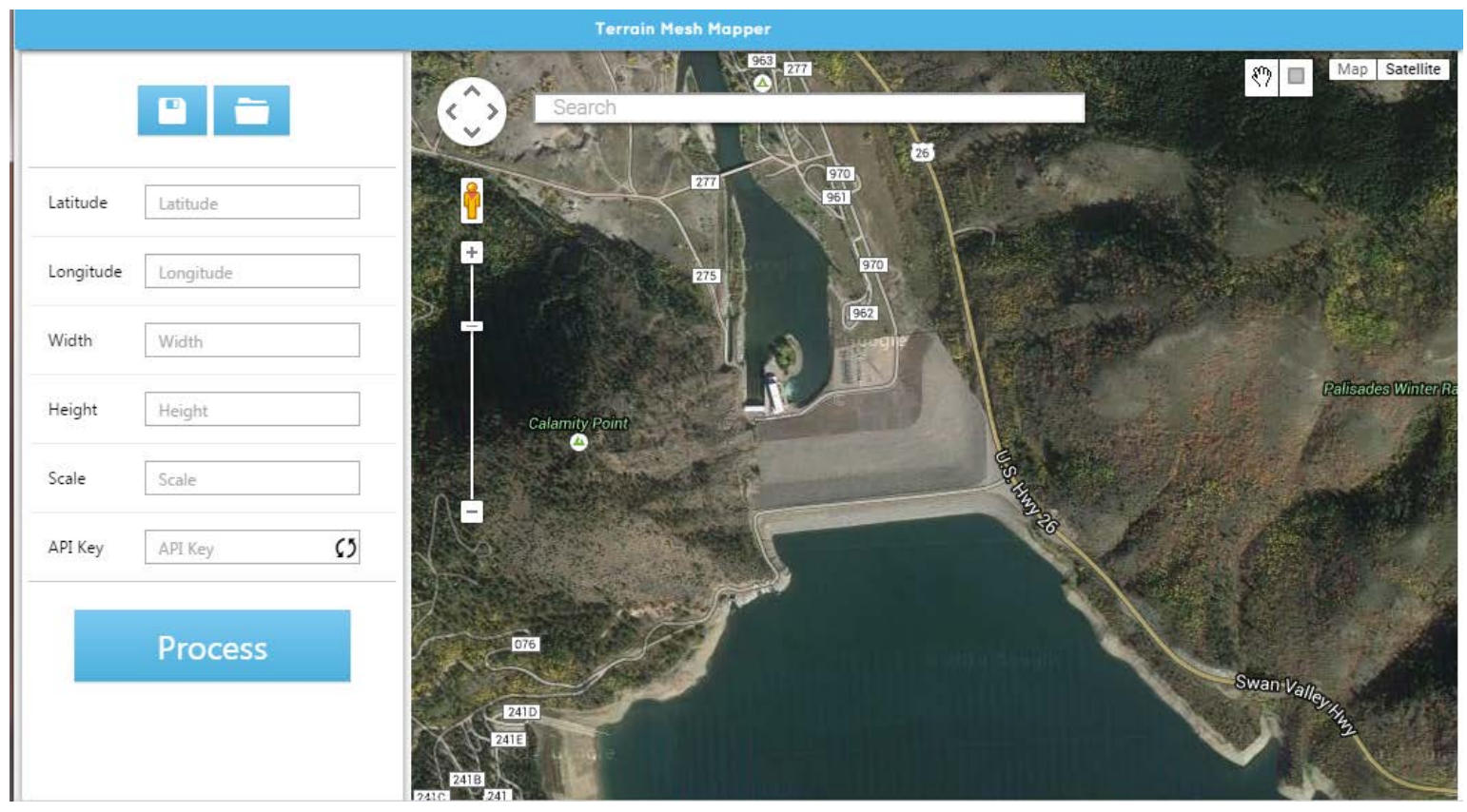

Figure 9: User interface for the Terrain Mesh Mapping tool.

\subsubsection{Running the 3D Model}

Once the 3D models of the facility and site are completed, these may be used to represent different kinds of physics related to initiating events such as external hazards (e.g., seismic, flooding, high winds). While different kinds of physics "toolkits" are available for use, as part of the aSMR analyses, we have focused on flooding representation using a Smoothed Particle Hydrodynamics (SPH) based approach.

SPH is a common technique for simulating fluids using pairwise forces between particles. SPH has important potential benefits, such as the ability to handle complex boundaries, discontinuities, and smallscale phenomena. SPH was originally designed for solving astrophysical problems by using a mesh-free Lagrangian fluid simulation technique. Mesh-free means that the approach does not require a stationary grid when solving fluid equations of motion - this is in contrast to Eulerian techniques which require an underlying grid. SPH works by obtaining approximate numerical solutions of the equations of fluid dynamics by representing the fluid with particles, where the physical properties and equations of motion of these particles are based on the continuum equations of fluid dynamics. Further, physical quantities are estimated by interpolating existing fluid quantities using the neighboring particles.

Example of flooding analysis using a SPH-based flooding module is illustrated in Figure 10 where we show the actual particles that represent the external flood initiating event. While this event was representative of a tsunami, the SPH approach is applicable for a variety of other flooding events such as internal floods (e.g., pipe break, inadvertent draining), large precipitation, dam breaks, river rises, etc. 


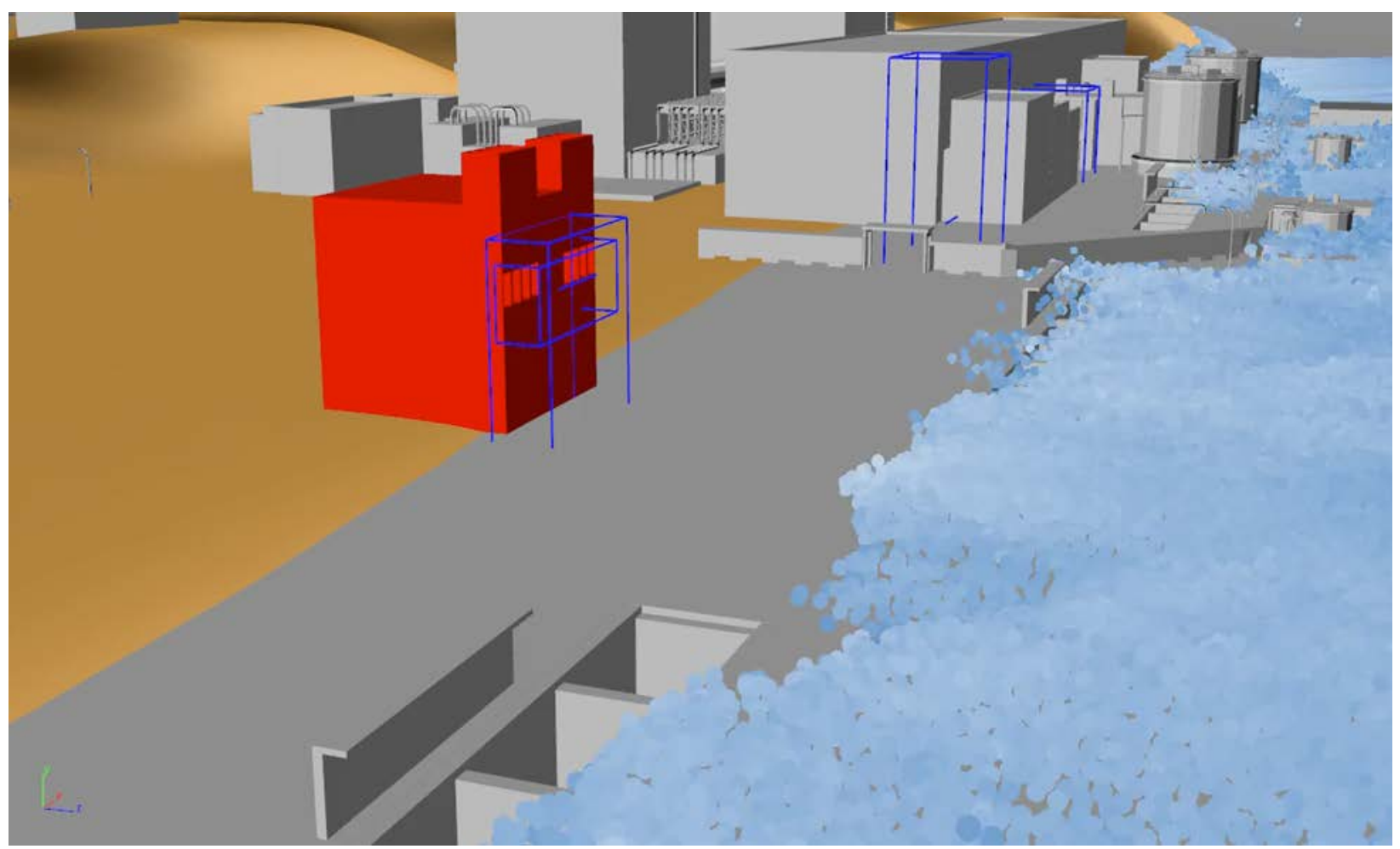

Figure 10. SPH-based flooding example showing an external flood potentially impacting facility infrastructure.

In addition to the visual representation of scenarios like the tsunami flooding, a variety of physicsbased information is available. For example, in Figure 11, we see that the loading force on the building is identified as part of the scenario simulation. This force can then be used to determine if the structure itself fails or if parts of the structure fails (or are impacted) such as doors and windows. Additional information such as the height of the water on the building (see Figure 12) is also available from the data that is stored in the simulation. 


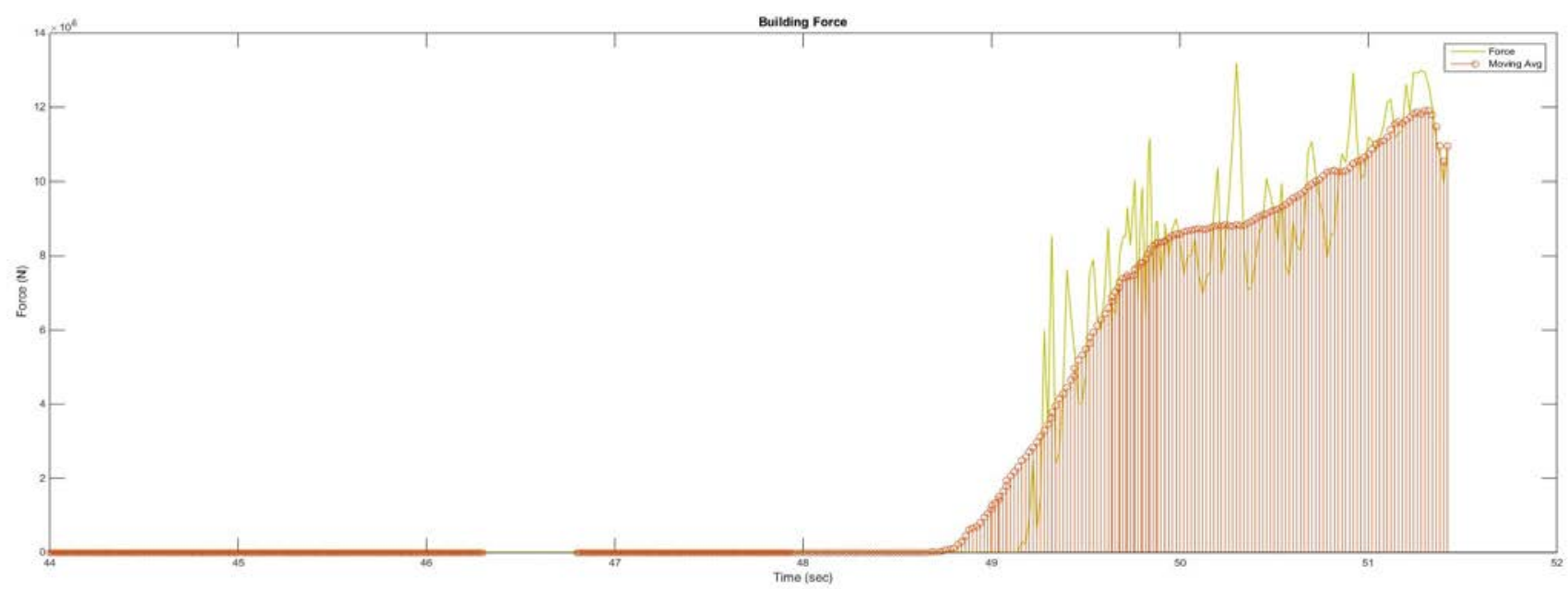

Figure 11. Force on the target facility infrastructure due to water impact.

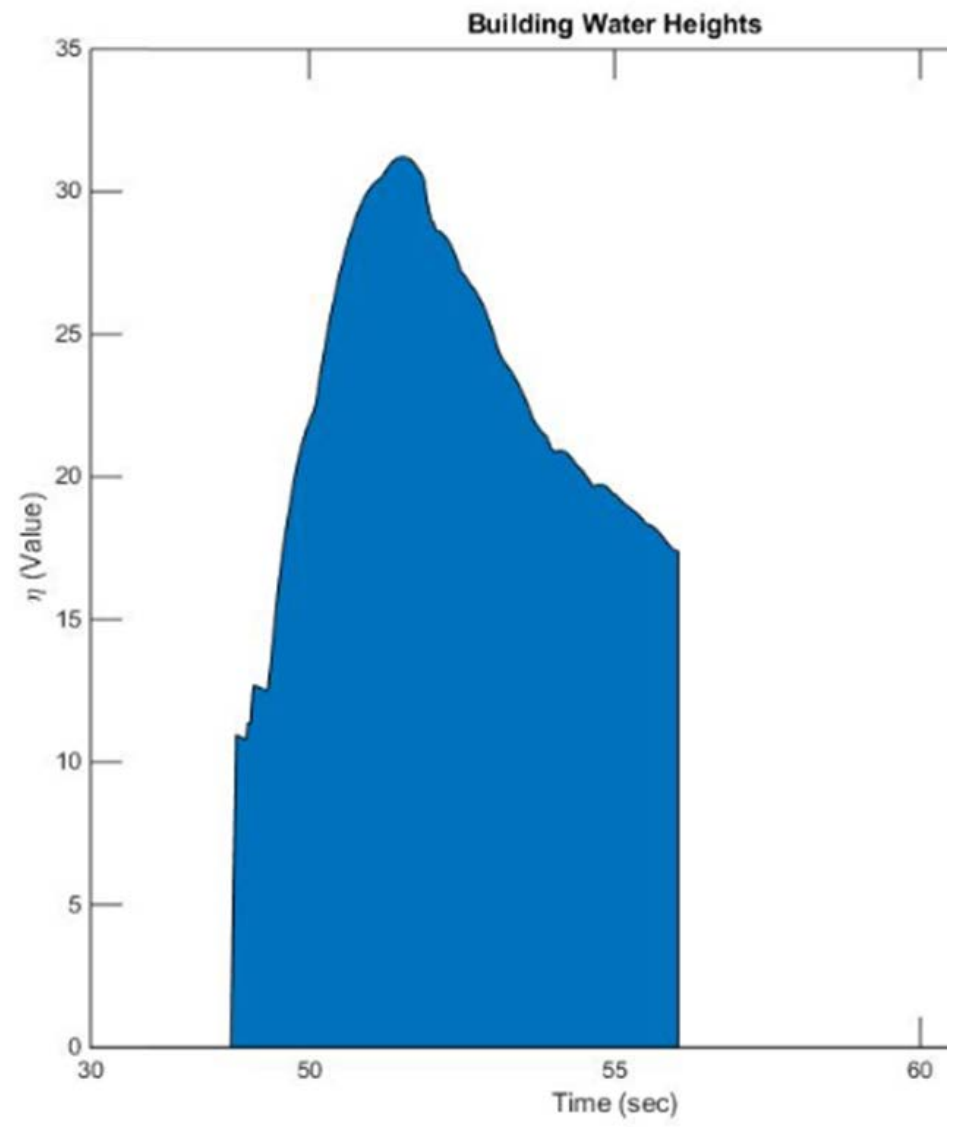

Figure 12. Height of water on the RCCS as a function of time during a tsunami scenario.

During a risk analysis simulation, multiple different initiating events are run, for example to represent different magnitudes of potential floods (e.g., tsunami heights). For each scenario though, the simulation is allowed to progress (for example, see Figure 13) to represent a sequential string of events. During the simulation, some events are represented probabilistically - consequently, outcomes from 
similar scenarios may differ due to the stochastic nature of the scenario. The most common probabilistic events in these types of physics-based scenarios is for the determination of component or structure failures. For example, during one simulation, a door that is inundated with water may fail, thereby allowing water to enter a building while another similar scenario may result in the door not failing.
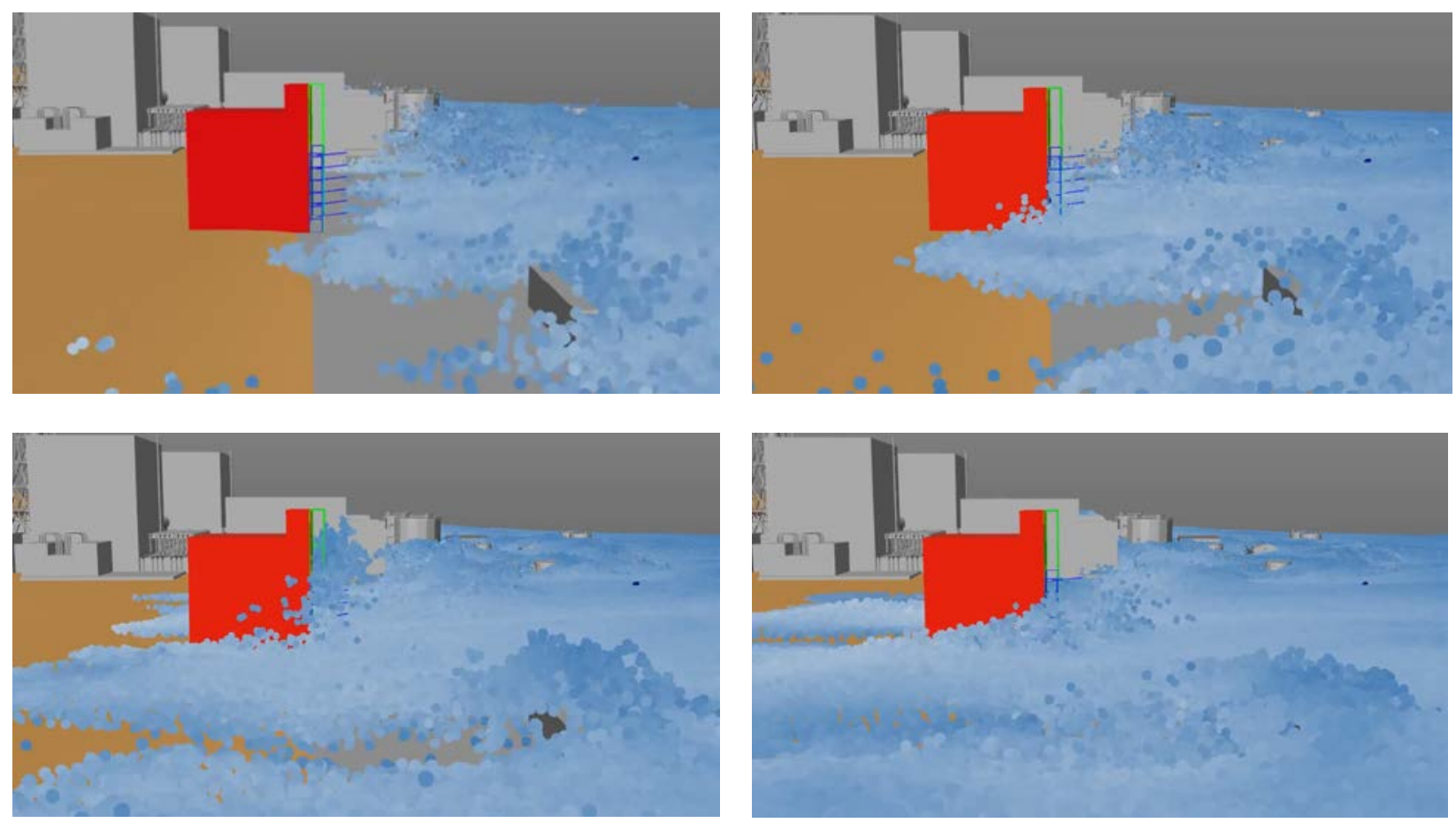

Figure 13. Progression of water on the RCCS during a tsunami scenario simulated using the 3D model and water physics.

\subsubsection{Debris Representation}

To simulate debris, for example as part of a flooding scenario, rigid objects need to be coupled to a fluid simulation tracking with the fluid and providing boundary conditions for the fluid as well. While the two-way coupling of particle-based fluids and solids seems to be straightforward, there is no general agreement how this should be handled. On one hand, the coupling has to cope with particle deficiency issues at the boundary in order to prevent spatial and temporal discontinuities of physical properties of the particles and sticking artifacts (3) The challenges of simulating a coupled flow with SPH have been investigated and are described in this section.

In fluid simulation, the interesting fluid behavior usually emerges when rigid objects are added to a simulation. A momentum-conserving two-way coupling method for SPH fluids and arbitrary rigid objects that is completely based on hydrodynamic forces is needed as well as to account for the moving boundary conditions arising as a result of the coupled flow. To solve these issues, we model the sur face of rigid bodies with "boundary particles" that interact with the fluid, thereby preventing deficiency issues and both spatial and temporal discontinuities. The problem of inhomogeneous boundary sampling is 
addressed by considering the relative contribution of a boundary particle to a physical quantity. This "boundary particle" approach facilitates not only the initialization process of the SPH calculation but also allows the simulation of multiple dynamic objects. Consequently, thin structures consisting of only one layer or one line of boundary particles, and also non-manifold geometries can be handled without any additional treatment.

The density summation approach approximates the density of a fluid particle correctly only if a particle is spherically surrounded by particles with the same initial density. Therefore, densities of fluid particles near the boundaries may be underestimated. In order to alleviate this underestimation, we set the densities of such particles to the rest density of the fluid. Even though this simple correction scheme significantly improves the situation, the density gradient still remains discontinuous near the boundaries. Additionally, since the particles near the boundaries do not have neighbors that spherically surround them, forces on such particles constrain their movements to the boundaries, which cause some "sticking" artifacts. To minimize this problem, we take the neighboring boundary particles into account when computing densities and forces for fluid particles.

For our debris representation, the particles that are attached to the rigid bodies are computed either directly (e.g. for analytical shapes) or from mesh representations. At regions with high-curvature, the particle distribution may be non-homogenous, resulting in a denser sampling in such areas. An example of objects with high-curvature would be the tank object shown in Figure 14.
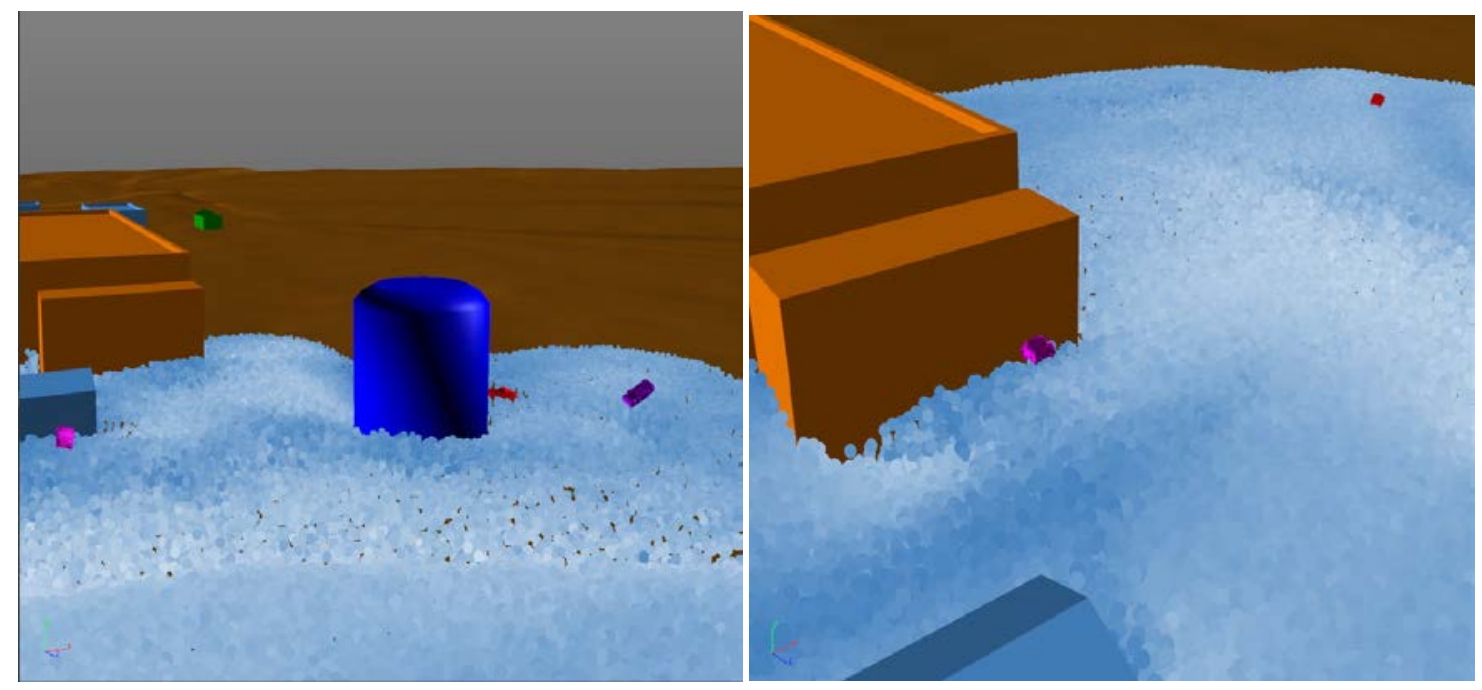

Figure 14. Representation of rigid bodies and debris in the SPH flood simulation.

Since the homogeneity of rigid body-to-particle sampling is not guaranteed, a result is that fluid particles may provide overly-large contributions to density/force calculations from the sampled regions. Those overestimated densities cause large pressure forces and therefore stability issues. This issue is due to the fact that the contribution of boundary particles does not consider the volume of a particle. This contradicts with the SPH concept, where the contribution of a particle in the approximation of any field variable should be governed by its volume. Therefore, we adjust the contribution of a boundary particle to a fluid particle by taking the volume of the boundary particle into account as a corrected density term. Since for SPH simulations we are using kernels, the weight of the next layer of particles is lower compared to the boundary layer, thus this issue quickly diminishes as a function of position. Therefore, using a single layer of boundary particles and taking the missing particles into account is a decent approximation in practice. 
To test the SPH physics module for a flooding risk calculation, we created a hypothetical plant model. The plant represented a typical site with a nearby lake adjacent to it, where the lake was contained via a earthen dam. We used the Terrain Mesh Mapping tool to create a plant site ground topography file as part of the 3D model creation. Then, for the lake, we filled the lake region with fluid particles by converting the container into a volume (up to the presumed level of the lake) and filling particles with it.

To represent an initiating event, we presumed a postulated breach of the earthen dam. The actual breach of the dam was modeled as a separate geometry and at a specific point in time was removed to initiate the hypothetical breach (see Figure 15). A close up of the scenario will reveal that a variety of objects are populated in the 3D model and these objects are allowed to move due to the rigid body-SPH particle physics interactions. For example, as shown in Figure 16, we place debris (truck) objects on the plant site such that they may be moved by the force of the SPH-based water.
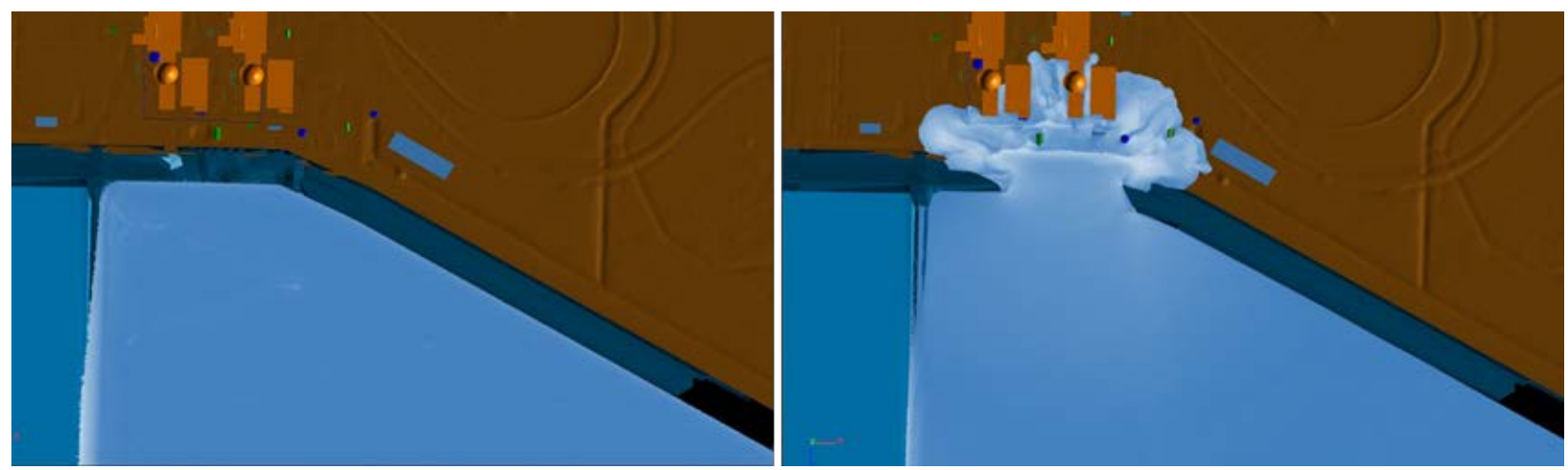

Figure 15. Example of the dam breach scenario simulation.

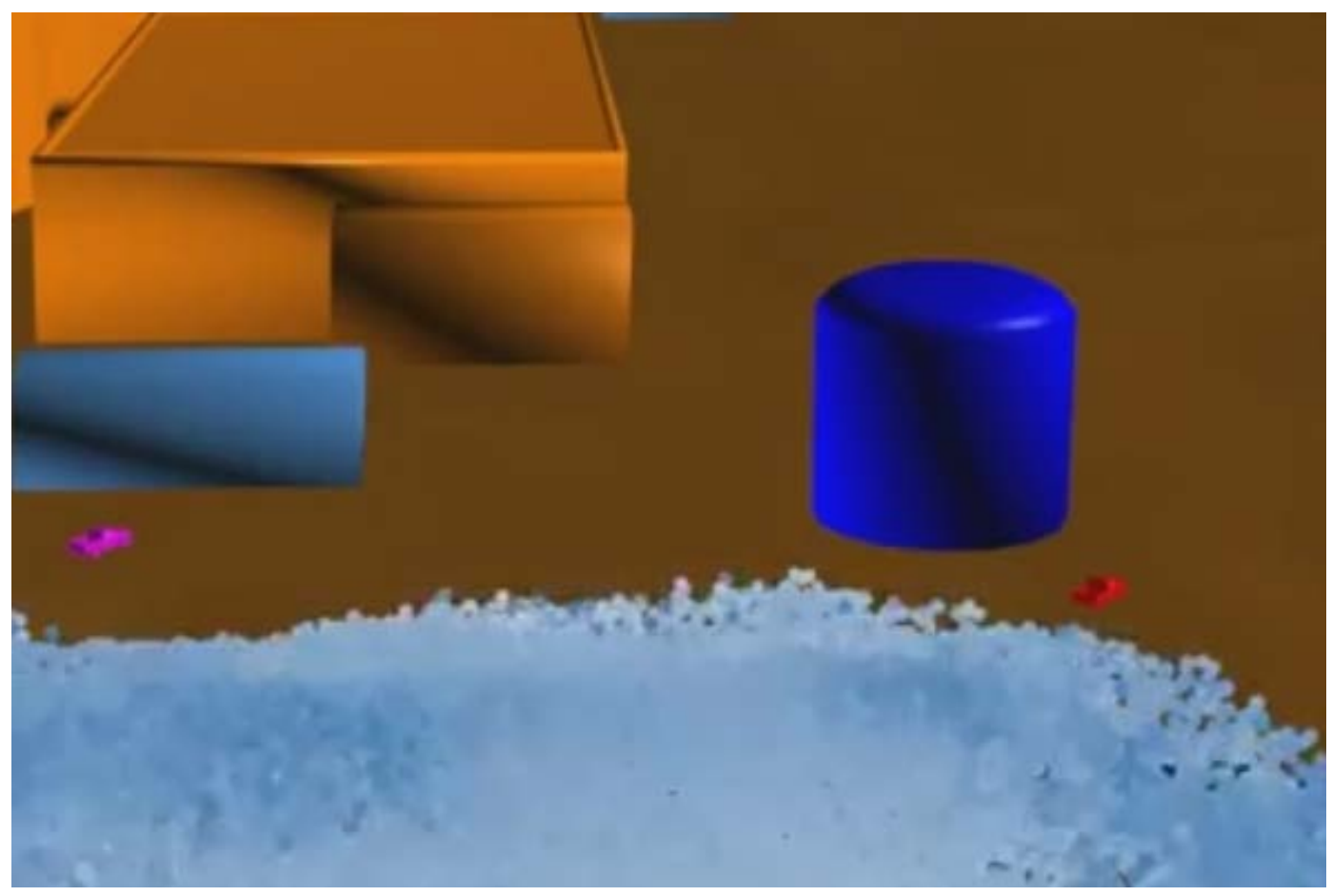

Figure 16. SPH flood calculation showing the debris (truck) objects that may be moved during the simulation by the force of the water. 
Lastly, as part of the simulation, we also keep track of forces imparted on and by objects and the water. Figure 17 shows that very detailed information is available for physics-based information such as the water velocity (the scale shown at the bottom of the figure), forces on the solid bodies (the scale shown at the left of the figure), and elevation information (the scale shown at the right of the figure).

By combining two types of physics, SPH-based water particles with solid-body objects, we have successfully demonstrated the ability to represent complex risk analysis scenarios that include debris representation.

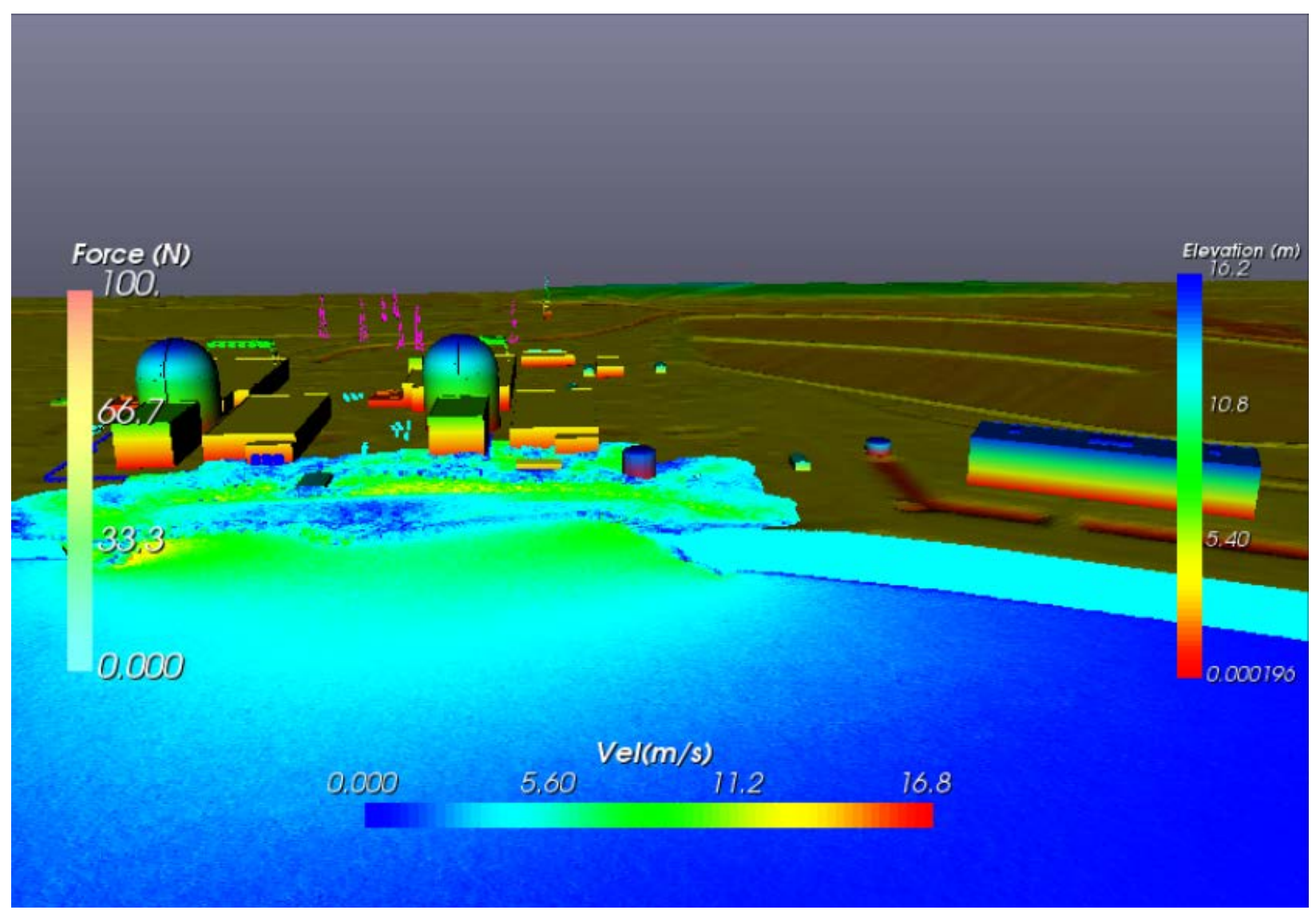

Figure 17. Example of the force and velocity information that is available to analysts as part of the flooding simulation. 


\section{CONCLUSIONS}

The goal of the advanced SMR PRA activity has been to develop quantitative methods and tools and the associated analysis framework for assessing a variety of risks. These risks will be focused on advanced SMR designs and operational strategies as they relate to the technical basis behind safety characterization.

Development and implementation of advanced SMR safety assessment methods may require new analytic methods or adaptation of traditional methods to the advanced design and operational features of these SMRs. By moving beyond the current limitations of static, logic-based models, we aim to provide integrated, scenario-based models based upon predictive modeling. The development of advanced SMRspecific safety models for margin determination will provide a safety case that describes potential accidents, design options (including postulated controls), and supports licensing activities by providing a technical basis for the facility safety envelope.

In this document, we provided the overall status of the work performed to expand and advance the state-of-the-practice in PRA. This work has focused on developing a framework for applying modern

computational tools to create advanced risk-based methods for identifying design vulnerabilities in SMRs. This framework has resulted in the fusion of state-of-the-art PRA methods and advanced 3D modeling methods. 


\section{REFERENCES}

1. Smith, C., et al., et al. Small Modular Reactor (SMR) Probabilistic Risk Assessment (PRA) Detailed Technical Framework Specification. s.1. : Idaho National Laboratory, 2013. INL/EXT-13-28974.

2. Prescott, S., et al., et al. Framework Development Supporting the Safety Portal. s.1. : Idaho National Laboratory, 2015. INL/EXT-15-35912.

3. Boundary handling and adaptive time-stepping for PCISPH. Ihmsen, M., et al., et al. s.1. : Virtual Reality Interaction and Physical Simulation, 2010, Vol. 2010, pp. 79-88. Proceedings for VRIPHYS 2010. 